\title{
Kyste osseux solitaire chez un patient atteint d'une drépanocytose
}

\section{Clinical diagnosis of solitary bone cyst in a patient with sickle-cell anemia}

\author{
SAID NOUJ AIM*, ALP ALANTAR*, J EAN-MARC GALEAZZI*, PHILIPPE GENET**, LOUIS MAMAN***
}

\begin{abstract}
RÉSUMÉ
Un patient âgé de 28 ans, d'origine africaine, est hospitalisé en urgence pour une crise drépanocytaire aiguë. L'examen clinique révèle un bon état général. II est adressé au service d'Odontologie pour une tuméfaction palatine, apparue une semaine auparavant. Une restauration en composite sur 21 et une carie de l'émail sur 22 sont notées. Les tests de vitalité au froid sont négatifs sur ces deux dents. La radiographie rétro-alvéolaire révèle une image radioclaire au contact des apex de 21,22 et 23 , sans continuité avec la lamina dura. La radiographie occlusale du maxillaire montre une lésion radioclaire para-médiane gauche, mesurant $20 \times 20 \mathrm{~mm}$, à limites nettes. Le diagnostic préliminaire évoqué est un abcès sous-périosté d'origine dentaire en rapport avec 21 et 22. Le traitement endodontique est réalisé dans un premier temps. L'exérèse de la lésion est effectuée sous anesthésie locale après la réalisation d'un lambeau vestibulaire de pleine épaisseur, l'ostéotomie de la corticale vestibulaire met en évidence une cavité osseuse vide, contenant un peu de liquide d'aspect hématique. Les racines de 21 et 22 plongent dans cette cavité qui jouxte la racine de 23 . Après aspiration du liquide, aucun revêtement épithélial n'est trouvé et le diagnostic de kyste osseux solitaire est retenu. Après la régularisation des rebords osseux alvéolaires, la suture est réalisée avec des points simples et séparés. Aucune suite opératoire notoire n'est rapportée. Les clichés radiographiques rétro-alvéolaires et les incidences occlusales sont pratiqués à un mois et à 14 mois postopératoires ; ces derniers montrent une réossification totale de la lacune osseuse. L'association d'un kyste osseux solitaire à une drépanocytose peut être expliquée par l'aptitude de ces patients à développer des infarctus osseux. La diminution de la vascularisation est un des mécanismes retenus dans la genèse de ces kystes. Ce cas permet de rappeler l'importance des bilans de recherche de foyers infectieux et la prévention des complications infectieuses post-chirurgicales chez les patients atteints d'une drépanocytose. (Med Buccale Chir Buccale 2003; 9: 105-111)
\end{abstract}

mots clés : Drépanocytose, Physiopathologie, Epidémiologie, Kyste osseux solitaire, Chirurgie buccale, Complications.

\section{SUMMARY}

A case of a solitary bone cyst in a patient with a sickle-cell anemia is reported. The patient was a 28-year-old black african male with a painfull palatal swelling. The physical examination was normal and the $\mathrm{X}$-rays showed a periapical radiolucency arround 21,22 and 23 without significant thermic tests. A diagnosis of dental abcess was done and endodontic treatment was firstly performed. Then during surgical exploration, using a buccal mucoperiosteal flap, a large endosseous hematic cavity $(20 \times 20 \mathrm{~mm})$ was found. Since this bone cavity was empty without demonstrable associated membrane or tissue, a clinical diagnosis of hemorrhagic bone cyst was made. No operative complication was noted an a complete bone healing occured after 14 months. The association of an hemorrhagic bone cyst with a sickle-cell anemia is discussed. The high frequency of bone infarction in patients with sickle-cell anemia may be an explanation. According to this case report, the importance of the prevention of oral infections in patients with sickle-cell disease is underlined. (Med Buccale Chir Buccale 2003; 9: 105-111)

key words : Anemia, Sickle-cell disease, Physiopathology, Epidemiology, Solitary Bone Cyst, Oral surgery, Complications.

* Service d'Odontologie, Centre Hospitalier de Nanterre, 92000 Nanterre, France

* Service d'Hématologie du Centre hospitalier Victor Dupouy, 95107 Argenteuil, France

* U.F.R d'Odontologie de l'Université Paris 5-René Descartes, 92120 Montrouge, France

Demande de tirés à part :

Saïd Noujaim, Centre Hospitalier de Nanterre, Service d'Odontologie, 403 avenue de la République, 92014 Nanterre, France Article reçu le 11 juillet 2002. Accepté pour publication le 18 mars 2003.

Article publié par EDP Sciences et disponible sur le site http://www.mbcb-journal.org ou http://dx.doi.org/10.1051/mbcb/2003006 
médecine buccale chirurgie buccale
La drépanocytose concerne l'odontologiste par certaines de ses manifestations (pâleur des muqueuses, retard d'éruption des dents, dystrophie des os plats), ses complications (foyers de thrombose et d'infection) et les mesures préventives qui doivent être prises pour toute intervention de chirurgie buccale [1]. L'observation clinique d'un kyste osseux solitaire dans la région de 21 et 22 découvert chez un patient hospitalisé pour une crise drépanocytaire aiguë est présentée. Le traitement radiculaire de ces deux dents a précédé l'exérèse de la lésion sous antibiothérapie. Le contrôle radiographique au $14^{\mathrm{e}}$ mois postopératoire montre une cicatrisation osseuse complète. Les mesures préventives pour la chirurgie buccale chez le patient drépanocytaire et l'association d'un kyste osseux solitaire à une drépanocytose sont discutées.

\section{OBSERVATION}

Un patient âgé de 28 ans, d'origine africaine, est hospitalisé en urgence pour une crise drépanocytaire aiguë qui ne cède pas sous oxygène nasal et dextropropoxyphène. II est adressé au Service d'Odontologie de l'Hôpital de Nanterre pour une tuméfaction palatine douloureuse.

Les antécédents associent une drépanocytose homozygote [SS], une appendicectomie, une cholécystectomie, une occlusion sur bride et plu-

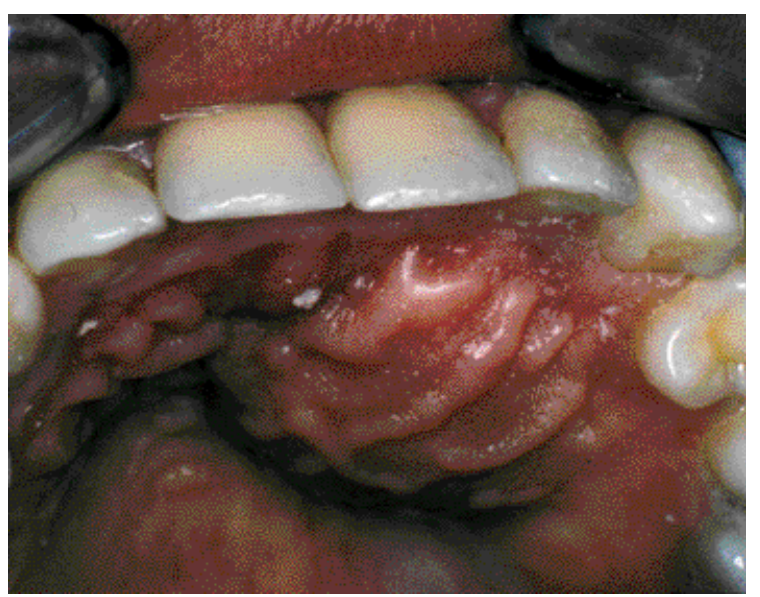

Figure 1 : Aspect faisant évoquer un abcès palatin sous-périosté.

Palatal swelling. sieurs hospitalisations répétées pour des crises drépanocytaires.

L'examen clinique révèle un bon état général, avec un pouls à 88 pulsations/minutes, une pression artérielle de $130 / 80 \mathrm{~mm} \mathrm{Hg}$ et une température corporelle de $37,7^{\circ} \mathrm{C}$. Le tableau clinique est dominé par des algies diffuses, thoraciques et des membres inférieurs, sans déficit neurologique. La muqueuse buccale a un aspect normal. A l'interrogatoire, le patient se plaint d'une «grosseur» dans la région palatine, douloureuse, apparue une semaine auparavant, et entraînant une gêne fonctionnelle. Aucune notion de choc sur les incisives n'est retrouvé.

L'examen endo-buccal révèle une tuméfaction antérieure gauche, dépressible, mesurant $20 \times 20 \mathrm{~mm}$ (Fig. 1). Le test de vitalité au froid est négatif sur 21 et 22 , positif sur 23 . Les examens radiographiques rétro-alvéolaire (Fig. 2), panoramique et occlusal (Fig. 3) révèlent une image radioclaire maxillaire bien limitée, sans continuité avec la lamina dura de 21 et 22 , venant au contact de la 23.

Le traitement endodontique et l'obturation radiculaire de 21 et 22 sont réalisés sous antibiothérapie (Amoxicilline 2g.j ${ }^{-1}, 7$ j). Comme la 23, dont la racine jouxte la cavité, répond positivement au test de vitalité au froid, la vitalité de cette dent est conservée et sera soumise à un surveillance régulière. Etant donné la taille de la lésion, son exérèse

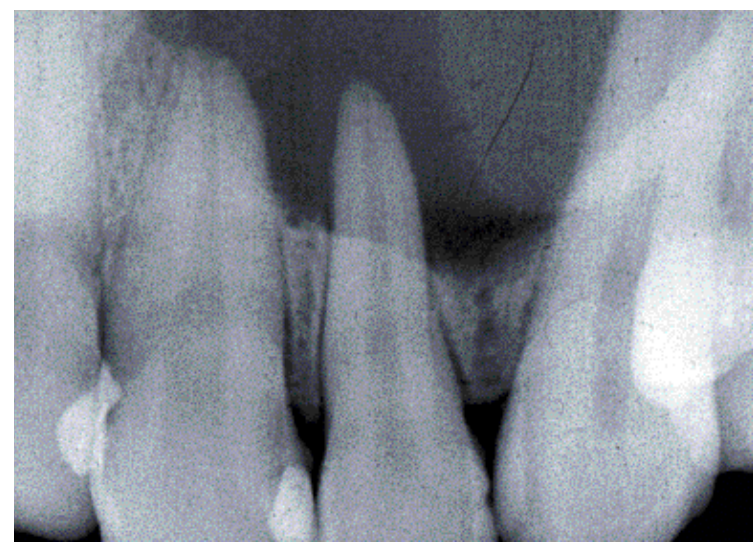

Figure 2 : Sur la radiographie rétro-alvéolaire, on découvre une large image radioclaire centrée sur la racine de 22 sans continuité avec la lamina dura. Periapical $x$-ray showing a radiolucency. 


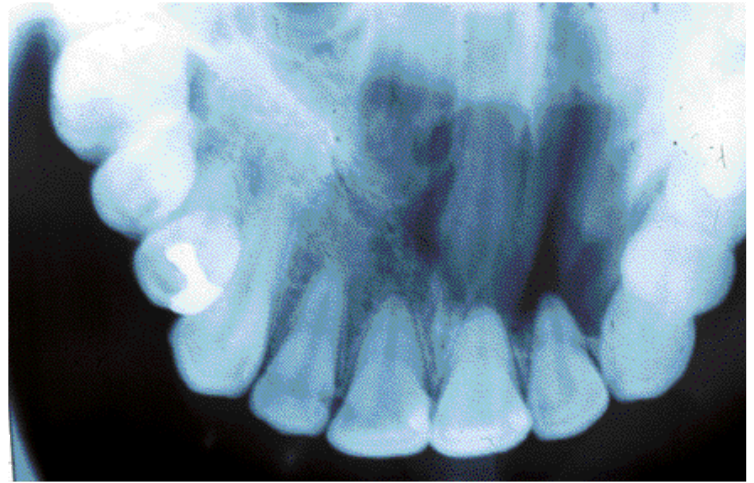

Figure 3 : Sur le cliché occlusal, l'image radioclaire étendue bien limitée semble en contact au moins avec trois apex.

Occlusal x-rays.

chirurgicale sous anesthésie locale est programmée une semaine après le traitement endodontique.

- Bilan biologique d'entrée

- Numération Formule Sanguine :

- Leucocytes : 17 300. $\mathrm{mm}^{-3}$

- Lymphocytes : 5 070. $\mathrm{mm}^{-3}$

- Polynucléaires : 10 950. $\mathrm{mm}^{-3}$

- Plaquettes : 312 000. $\mathrm{mm}^{-3}$

- LDH : $1241 \mathrm{U.}^{-1}$

- Hémoglobine : 8,3 gr.100. $\mathrm{ml}^{-1}$

- Cytolyse modérée

- lonogramme sanguin :

- Sodium : $134 \mathrm{mmol.}^{-1}$

- Potassium : 4,15 mmol..$^{-1}$

- Urée : 5,10 mmol..$^{1}$

- Créatinine : $59 \mu \mathrm{mol} . \mathrm{I}^{1}$

- Vitesse de sédimentation : $38 \mathrm{~mm}$ à la première heure

- Hémocultures négatives

- ECBU stérile

- Evolution et traitement de la crise dépranocytaire :

- Amendement de la symptomatologie douloureuse sous buprénorphine SC puis sulfate de morphine (60 mgX2. $\mathrm{j}^{-1}$ ) avec du paracétamol codéïné en relais,

- Régression du syndrome fébrile sous ciprofloxacine (500 mgX2. $\mathrm{j}^{-1}$ ),

- Apparition d'un globe vésical sous morphine ayant nécessité un sondage urétral pendant 12 jours.

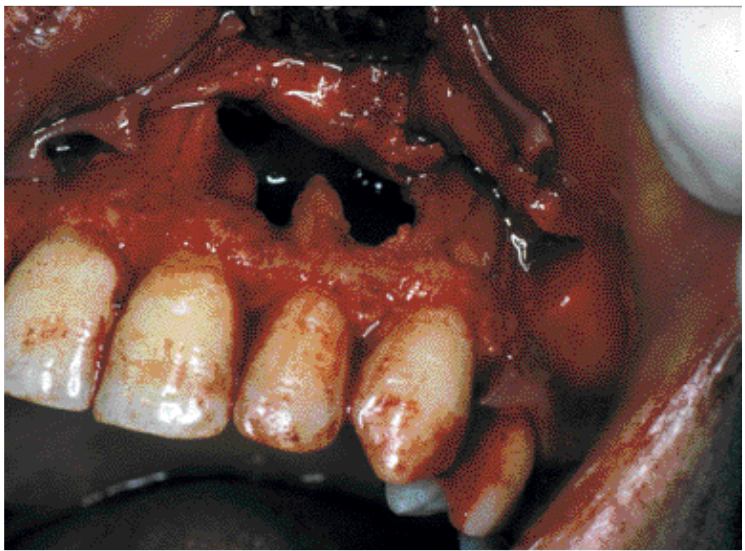

Figure 4 : Vue per-opératoire.

Surgical procedure.

- Prescription pour l'intervention : un relais avec amoxicilline : $2 \mathrm{~g} . \mathrm{j}^{-1}$ pendant 8 jours après le traitement radiculaire est institué.

- Intervention chirurgicale :

- L'anesthésie est pratiquée aux deux foramina infra-orbitaires avec un rappel au foramen rétro-incisif (articaiine adrénalinée, 1/200.000).

- L'incision intra-sulculaire vestibulaire s'étend de 12 à 23 avec double décharge distale. Un lambeau de pleine épaisseur et une trépanation de la corticale vestibulaire mettent en évidence une cavité osseuse vide mesurant $20 \times 20 \mathrm{~mm}$, dans laquelle plongent les apex de 21 et 22 , et qui vient au contact du bord mésial de la racine de 23 (Fig. 4). L'aspiration de liquide intra-cavitaire de type hématique est complétée par l'exploration de la cavité à la curette de Lucas : elle révèle une large résorption de l'os palatin permettant un contact avec la muqueuse palatine sur toute la surface de la tuméfaction palatine. Les rebords osseux alvéolaires sont régularisés avant la réalisation de la suture avec des points simples et séparés.

- Prescription : poursuite de l'antibiothérapie, paracétamol à la demande, et bain de bouche à la thyrothricine pendant six jours.

- Les sutures sont déposées au 7e jour. Aucune suite opératoire n'est rapportée.

- Sortie du patient 25 jours après l'intervention avec une ordonnance :

- Acide folique $10 \mathrm{mg} . \mathrm{j}^{-1}$, paracétamol 2 g.j ${ }^{-1}$ médecine buccale chirurgie buccale VOL. $9, \mathrm{~N}^{\circ}$ 2003 page 107 


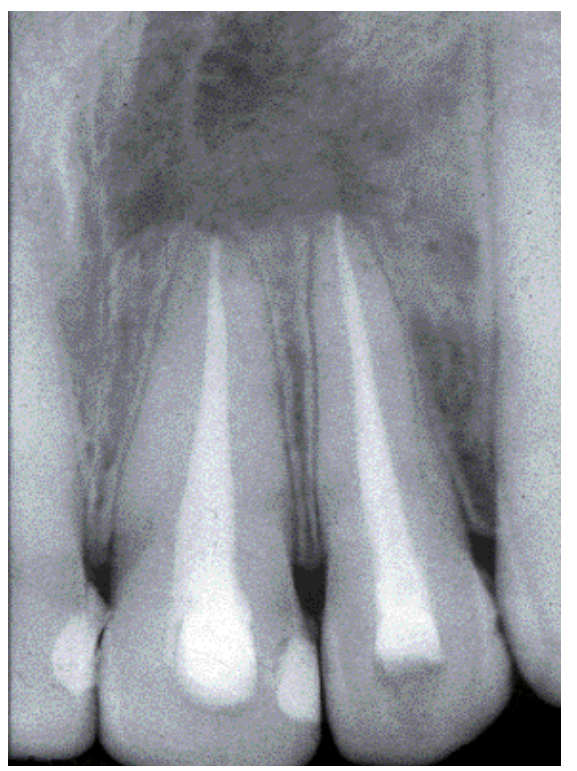

Figure 5 : Cliché retro-alvéolaire de contrôle au $14^{\mathrm{e}}$ mois post-opératoire : la réossification a complètement comblé la cavité.

médecine buccale chirurgie buccale

L. $9, \mathrm{~N}^{\circ} 2$

2003

page 108
Evolution du kyste osseux solitaire :

Un contrôle au $1^{\text {er }}$ mois puis au $14^{\mathrm{e}}$ mois postopératoires (Fig. 5) est réalisé. A chaque contrôle, des clichés rétro-alvéolaires et une incidence occlusale sont pris et un test au froid réalisé sur 23 pour vérifier la vitalité pulpaire.

Les clichés montrent que la réossification de la géode osseuse est totale après 14 mois. La sensibilité pulpaire de 23 est normale.

\section{DISCUSSION \\ Epidémiologie et pathogénie de la drépanocytose}

La drépanocytose survient presque exclusivement dans les populations d'Afrique équatoriale et tropicale - et les populations qui en sont issues (Antilles, Etats-Unis) - de I'Inde, de Madagascar [1-3] et d'Asie du Sud-Est pour certaines formes. A un degré moindre, les populations du pourtour méditerranéen peuvent être atteintes.

Chez l'adulte sain, les molécules d'hémoglobine sont constituées de 2 chaînes de globine $\alpha$ et de 2 chaînes de globine $\beta$, conduisant à la synthèse d'une hémoglobine appelée $\mathrm{HbA}$. La drépanocytose, ou anémie à hématies falciformes, est une maladie génétique, autosomale, récessive, due à la synthèse d'une hémoglobine anormale (hémoglobine $\mathrm{S}$ ou $\mathrm{HbS}$ ). Cette synthèse est secondaire à une mutation sur le $6^{e}$ codon du gène $\beta$ de la globine aboutissant à la substitution de l'acide glutamique par une valine. Ce changement d'acide aminé entraîne, en particulier lors des états d'hypoxie, une polymérisation de I'hémoglobine $S$ qui va aboutir entre autres à une déformation des globules rouges (hématies falciformes en raison de leur forme de faux). Les conséquences de ce phénomène sont nombreuses, une des plus importantes étant l'occlusion des vaisseaux de petit calibre à l'origine d'une anoxie en aval se traduisant par les classiques crises vaso-occlusives [1].

Seuls les sujets homozygotes [SS] présentent des symptômes, les sujets hétérozygotes [AS] sont asymptomatiques. Le trait drépanocytaire peut être associé à d'autres anomalies de l'hémoglobine ( $\beta$-thalassémie, $\mathrm{HbC}, \mathrm{HbD}, \mathrm{HbE} \ldots$ ). Certains patients doubles hétérozygotes, en particulier ceux qui sont [SC], sont symptomatiques [1].

Le diagnostic biologique est fait par l'électrophorèse de l'hémoglobine. La drépanocytose s'accompagne d'une anémie normochrome, normocytaire avec un taux d'hémoglobine qui peut s'abaisser jusqu'à $6 \mathrm{~g} .100 \mathrm{ml}^{-1}[1]$. Les conséquences cliniques vont être :

- Une anémie chronique, parfois aggravée par certains phénomènes intercurrents (carence notamment en folates, érythroblastopénie liée au parvovirus B 19...).

- Des infarctus osseux et viscéraux qui sont responsables des douleurs de la crise drépanocytaire aiguë et dont la répétition peut entraîner des lésions irréversibles dans certains organes: ostéonécrose de hanche, altération des fonctions rénales, cardiaques ou pulmonaires, atteintes neurologiques...

- Une sensibilité accrue aux infections, en partie en raison de l'asplénie fonctionnelle secondaire aux infarctus spléniques. Les infections 
pulmonaires sont les plus fréquentes, puis ce sont les ostéites, les méningites et les infections à Salmonelles [3-8].

- Une pâleur de la muqueuse buccale secondaire à l'anémie chronique, un retard d'éruption dentaire, voire un retard de croissance si l'anémie chronique persiste, une hypoplasie d'émail, une hypominéralisation de la dentine, une hypercémentose et une ostéite de la mandibule $[2,9]$.

\section{Conduite à tenir en chirurgie buccale chez le patient atteint d'une drépano- cytose}

Le sujet drépanocytaire peut subir des interventions de chirurgie buccale en ambulatoire, sous anesthésie locale, dès lors que la maladie est stabilisée. L'utilisation de vasoconstricteur n'est pas contre-indiquée; c'est d'avantage le stress engendré par l'intervention qui est susceptible de provoquer des complications [2, 9-12]. Les crises drépanocytaires peuvent être déclenchées par des infections diverses, une déshydratation, une hypoxie, une acidose, des traumatismes, une anesthésie générale ou des extractions dentaires $[2,7,10,13]$. Pour une anesthésie générale, des mesures spécifiques doivent être prises pour prévenir une déshydratation, une hypoxie, une acidose et une infection. La sédation profonde en IV sera évitée. L'oxymètrie permet de reconnaître la survenue d'une acidose respiratoire et d'une hypoxie [2]. Les précautions en chirurgie buccale sont liées aux risques infectieux et imposent d'une part la recherche systématique des foyers infectieux et d'autre part une antibioprophylaxie opératoire [2]. Le protocole conseillé par Sanservere et coll. [6] est un protocole per os : pénicilline : $2 \mathrm{~g}$, $1 \mathrm{~h}$ avant l'intervention et $1 \mathrm{~g}, 6 \mathrm{~h}$ après l'intervention ; en cas d'allergie à la pénicilline : clindamycine $300 \mathrm{mg}$ per os $1 \mathrm{~h}$ avant l'intervention et $150 \mathrm{mg}, 6 \mathrm{~h}$ après l'intervention. Une transfusion sanguine n'est généralement pas nécessaire pour la chirurgie buccale mais, en présence d'une anémie chronique sévère, on doit s'efforcer de ramener le taux $d^{\prime} \mathrm{Hb}$ à $10 \mathrm{~g} .100 \mathrm{ml}^{-1}[2]$.

\section{Observation clinique}

La présence d'un kyste osseux solitaire en dehors de tout antécédent de traumatisme peut s'expliquer par la prédisposition de ces sujets à développer des infarctus osseux [2,3]. La présence d'un kyste osseux solitaire en rapport avec des apex dentaires est fréquente et le site maxillo-facial de prédilection est mandibulaire postérieur ${ }^{[14]}$; seuls $2 \%$ de ces pseudokystes sont retrouvés dans le maxillaire [15]. Un trouble de la sensibilité, voire une absence de sensibilité des dents concernées, est parfois observé, ce qui peut le faire confondre avec un kyste inflammatoire radiculo-dentaire [16,17]. Le cas présenté répond à trois $(1,3$ et 4$)$ des quatre critères cliniques de la définition des kystes osseux solitaires proposée par Rushton (1946) [18]: (1) cavité osseuse solitaire sans revêtement épithélial (2) absence de signes apparents d'infection (3) contenu liquidien sans tissu de granulation et (4) présence de parois osseuses. Ces critères de définition doivent être révisés puisque les kystes osseux solitaires peuvent bien qu'exceptionnellement, être symptomatiques (œdème, douleur, paresthésie) [14, 19-22]. Bien que la voie d'abord palatine de la chirurgie apicale soit parfois indiquée (lésion touchant l'incisive latérale en l'absence de signes d'appel vestibulaire) afin d'éviter une tunnélisation osseuse [23], la taille de cette lésion a fait choisir la voie d'abord vestibulaire. La simple ouverture de la cavité complétée du parage de la cavité est le traitement le plus communément admis $[14,17,24,25]$. La qualité du scellement apical et la valeur stratégique des dents concernées ont fait opté pour un traitement conservateur sans résection apicale [26]. Le très bon pronostic de ces lésions, après une durée moyenne de consolidation osseuse de 12 mois [27], est confirmé par ce cas clinique. Les kystes osseux solitaires récidivants, nécessitant une seconde intervention, sont exceptionnels $[16,28]$.

\section{Hypothèses étiopathogéniques du kyste osseux solitaire et de son asso- ciation avec la drépanocytose}

L'étiologie des kystes osseux solitaires n'est à ce jour pas connue. Parmi les théories propo- médecine buccale chirurgie buccale VOL. $9, \mathrm{~N}^{\circ}$ 2003 page 109 
médecine buccale chirurgie buccale

)L. $9, \mathrm{~N}^{\circ} 2$ 2003

page 110 sées, la théorie traumatique est la plus souvent retenue $[19,25]$. Selon cette théorie, un traumatisme ancien sur un os spongieux entouré d'os cortical entraînerait la formation d'un hématome intra-osseux qui pourrait donner naissance à une cavité vide. Or cette notion de traumatisme n'est retrouvée que dans $27 \%$ à $50 \%$ des cas $[14,28]$. Certaines hypothèses envisagent un trouble dystrophique (association avec la dysplasie osseuse floride ou avec une pathologie de développement), un processus dégénératif $[29,30]$ ou une perturbation du flux sanguin [31]. La théorie du processus dégénératif est étayée par les résultats histologiques de l'étude de Beasley ( $\mathrm{n}=30$ cas) [14].

L'examen des tissus mous recueillis dans la cavité révèle un tissu fibromyxomateux dans les zones de lyse osseuse. La présence de débris cellulaires en l'absence de tout tissu organisé semble confimer cette thèse dégénérative. D'autres théories font intervenir (1) une infection de bas grade (2) des anomalies vasculaires intra-osseuses (3) la dégénérescence de tumeurs intra-osseuses ou (4) une altération locale du métabolisme osseux [32].

Chez les sujets atteints d'une drépanocytose, l'étiopathogénie ou kyste osseux solitaire semble due à un un phénomène ischémique, éventuellement favorisé par une infection locale, et qui évolue parfois vers une ostéonécrose. Les kystes solitaires peuvent toucher

\section{RÉFÉRENCES}

1 - Schaison G. La drépanocytose, un exemple de maladie constitutionnelle de l'hémoglobine. Rev Stomatol Chir Maxillofac 1983; 84: 144-6.

2 - NtIMA-nSIEMI K, MBuila C, Bascoulard M, Lemasson $\mathrm{PH}, \mathrm{ABBAR} \mathrm{H}$. Patients drépanocytaires en stomatologie. Quelle prise en charge ? Rev Stomatol Chir Maxillofac 1997; 98: 382-6.

3 - Girard P, J eandot J , Quevauvilliers J , Perlemuter L. Dictionnaire médical du chirurgien dentiste Masson, Paris, 2000, pp 241-2.

4 - WAJ CMAN H, LABIE D. Aspects actuels de la biologie de la drépanocytose. Ann Méd Intern 1981; 132 : 568-94.

5 - Begue P, Quinet B. Drépanocytose de l'enfant. Encycl Méd Chir, Pédiatrie, 4080 A 20, 1985. tous les os du squelette, mais la localisation tibiale est de loin la plus fréquente. Les autres localisations par ordre décroissant de fréquence sont : (1) le cubitus (2) la hanche, l'humérus et les petits os des extrémités et (3) le fémur et le péroné [33]. Aucune localisation maxillaire ne semble avoir été rapportée à ce jour. Malgré cette difficulté de classification anatomo-pathologique, le kyste osseux solitaire est une entité anatomo-clinique bien identifiée appartenant au groupe des pseudokystes [32,34,35].

La chirurgie buccale chez le patient atteint de drépanocytose implique une antibioprophylaxie systématique. L'adjonction d'un vasoconstricteur à l'anesthésique local n'est pas contre-indiquée. L'anesthésie générale impose une prévention de l'anoxie, des mesures spécifiques doivent être prises pour prévenir une deshydratation, une hypoxie, une acidose et une infection. Dans tous les cas, la susceptibilité accrue de ces sujets à l'infection implique des contrôles cliniques et radiographiques réguliers à la recherche de foyers infectieux.

L'originalité de ce cas clinique repose sur l'association d'un kyste osseux solitaire à une drépanocytose mais aussi à sa situation maxillaire et à la symptomatologie associée amenant une révision des critères de définition de ces pseudokystes.
6 - Sansevere J J , Miller M. Management of the oral and maxillo-facial surgery patients with sickle cell disease and related hemoglobinopathies. J Oral Maxillofac Surg 1993; 51: 912-6.

7 - Bedos JP, Chevret S, Chastan CL, Geslin P, Regnier B. Epidemiological features of and risk factors for infection by streptococcus pneumonia strains with diminished susceptibility to penicillin: findings of a french survey. Clin Infect Dis 1996; 22: 63-72.

8 - De Monttalembert M. Prise en charge de la drépanocytose en 1996. CTS Necker Enfants Malades, Paris, Pédiatrie Pratique 1996: 5-7.

9 - Peppers GC, Davis V, Atkinson J C. Sickle cell anemia: a case report and litterature review. Clin Prev Dent 1992; 14: 5-9. 
10 - Finet B. Anémie falciforme : ses implications cliniques lors des soins dentaires. Rev Méd (Liège) 1995; 50: 430-2.

11 - Frush K., W ARE RE, K InNEY TR. Emergency department visits by children with sickle hemoglobinopathies. Factors associated with hospital admission. Pediatr Emergency Care 1999; 11: 9-12.

12 - Mohsen AF, El-Hazmi, Al-Swailem A, Warsy AS. Glucose-6-phosphate deshydrogenase deficiency and sickle cell genes in Bisha. J Trop Pediatr 1995; 41: 225-7.

13 - Buchanam GR, S mith SJ. Pneumococcal septicemia despite pneumococcal vaccine and prescription of penicillin prophylaxis in children with sickle cell anemia. Arch Pediatr Adolesc Med 1986; 140: 428-32.

14 - BeASLEY J D. Traumatic cyst of the jaws: report of 30cases. J Am Dent Ass 1976; 92: 145-52.

15 - Copete MA., Kawamata A., Langlais RP. Solitary bone cyst of the jaws: radiographic review of 44 cases. Oral Surg Oram Med Oral Pathol Oral Radiol Endod 1998; 85: 221-5.

16 - NeXton CW, ZUNT SL. Endodontic intervention in the traumatic bone cyst. J Endod 1987; 13: 405-8.

17 - RuIz-Hubard EE, HARRISON J W. Healing of a traumatic bone cyst after non surgical endodontic treatment. J Endod 1987; $13: 40-2$.

18 - Rushton MA. Solitary bone cysts in the mandible. $\mathrm{Br}$ Dent J 1946; 81: 37-49.

19 - HowE GL. Haemorrhagic cysts of the mandible. $\mathrm{Br}$ J Oral Surg 1965; 3: 55-91.

20 - Hansen LS, Sapone J, Sproat RC. Traumatic bone cyst of the jaw. Report of sixty-six cases. Oral Surg Oral Med Oral Pathol 1974; 37: 899-910.

21 - Goodstein DB, Himmelfarb R. Paresthesia and the traumatic bone cyst. Oral Surg Oral Med Oral Pathol 1976; 42: 442-6.

22 - HALL AM, O RTH D. The solitary bone cyst. Report of two cases. Oral Surg Oral Med Oral Pathol 1976; 42: 164-8.

23 - Alantar A, Missika P. Abord palatin de la résection apicale : revue de la littérature et présentation d'un cas. Rev Odontostomatol 1992; 21: 189-93.
24 - Blum T. Unusual bone cavities in the mandible : a report of three cases of traumatic bone cysts. J Am Dent Assoc 1932; 19: 281-301.

25 - Lebreton G. Traité de sémiologie et clinique odontostomatologique. Cdp, Paris, 1997, pp 223-68.

26 - Missika P, Machtou P, Ouvrard H. Conceptions et techniques actuelles du traitement chirurgical des lésions apicales. Actual Odontostomatol 1980; 130: 221-36.

27 - Huebner GR, Turlington EG. So-called traumatic (hemorrhagic) bone cysts of the jaws: review of the litterature and case report of two unusual cases. Oral Surg Oral Med Oral Pathol 1971; 31: 354-65.

28 - Vijayaraghavan K, Whitlock RiH. An unusual nine cases of haemorrhagic bone cyst. $\mathrm{Br}$ J Oral Surg 1975; 13: 64-72.

29 - KUROI M. Simple bone cyst of the jaw : review of the litterature and report of case. J Oral Surg 1980; 38: 456-9.

30 - Kaugars Ge, Cale AE. Traumatic bone cyst. Oral Surg Oral Med Oral Pathol 1987; 63: 318-24.

31 - GlOWACKI M, M RCINIAK W. Solitary bone cyst: etiology, diagnosis, therapy, clinical and radiologic evaluation of treatments results. Chir Narzadow Ruchu Ortop Pol, 1997; 62: 437-44.

32 - COHEN J. Etiology of simple bone cyst. J Bone J oint Surg 1970; 52A: 1493-7.

33 - Mbumbe-King A, Horma C, Balde I, Diane C, VINAND $P$. Drépanocytose et chirurgie. Notre expérience à l'hôpital pédiatrique d'Owendo. Chir Pediatr 1979; 20: 409-12.

34 - Mitchell DA, WARD-B ooth RP. A typical presentation of a solitary bone cyst. Int J Oral Surg 1984; 13: 25659.

35 - Cudia G, Gordeeff A, Panzoni E. Le kyste osseux solitaire. Une observation à double localisation mandibulaire et humérale. Rev Stomatol Chir Maxilofac 1987; 88: 31-5. médecine buccale chirurgie buccale VOL. $9, \mathrm{~N}^{\circ}$ 2003 page 111 